Volume 9, No.5, September - October 2020

International Journal of Advanced Trends in Computer Science and Engineering

Available Online at http://www.warse.org/IJATCSE/static/pdf/file/ijatcse221952020.pdf

https://doi.org/10.30534/ijatcse/2020/221952020

\title{
COVID-19 Tweets Textual Analytics Using Machine Learning Classification for Fear Sentiment
}

\author{
Gobind Simran Kaur Gahir ${ }^{1}$, Jignyasa B Sanghavi ${ }^{2}$ \\ ${ }^{1}$ M.Tech Student, Department of Computer Science, Shri Ramdeobaba College of Engineering and Management, Nagpur, \\ Maharashtra, India, gahirgj@rknec.edu \\ ${ }^{2}$ Assistant Professor, Department of Computer Science, Shri Ramdeobaba College of Engineering and Management, Nagpur, \\ Maharashtra, India, sanghavijb1@ rknec.edu
}

\begin{abstract}
The volume of COVID 19 microblogging messages is increasing exponentially with the popularity of COVID 19 microblogging services. With the huge number of messages seem in user interfaces, it obstruct user accessibility to useful information hide in disorganized, incomplete and unstructured text message. In order to increase user accessibility it present to aggregator related COVID-19 microblogging message into the cluster and automatically allocate them semantically meaningful labels. However, a typical features of COVID-19 microblogging messages is that they are much shorter than standard text document these messages provide insufficient terms of information for capturing semantic associations providing solution to this problem it suggest a novel framework for organizing unstructured COVID-19 microblogging messages by transforming them into semantically structured representation it express informative tree fragments by analyzing a parse tree of the messages, and then utilize external knowledge bases to increase their semantic information. Twitter dataset shows that our framework significantly outperforms existing stateof-the-art methods. This research provide insights into Corona virus fear sentiment progression, and outlines associated methods, implication, limitations and opportunities.
\end{abstract}

Key words: COVID 19 micro blogging, machine learning, sentiment analysis, text analysis, twitter.

\section{INTRODUCTION}

In the last few years, COVID-19 microblogging services such as twitter has gained great popularity among internet users. Within an enterprise, microblogging behavior is bounded by the main approach in our analysis suggests that users with strong local topical alignment popularity of online social networks like Facebook and Twitter are the first year of adaptation of the microblogging service by the company. Sentiment analysis services for machine learning API and tools stock market prediction is one of the most attractive research topics since the machine learning models with some basic data, open high low close volume, and added some more. Twitter is becoming an increasingly popular microblogging platform transforming natural language text into numerical vectors is an important preprocessing step for many text mining tasks, such as cluster analysis and fear sentiment progression classification. Social media, specifically its microblogging platform twitter, serves as an ideal medium to give this understanding twitter has more than 145 million daily active users and allows people to post, repost, like, and comment on 'tweets' of up to 280 characters. This is timely since there was a large measles outbreak in the United States topics in the study of public opinion and sentiment. The outbreak of the novel coronavirus disease (COVID-19) has greatly (e.g., lockdown, social distancing). When zooming into the topiclevel analysis, we find that different determine the sentiment polarity of each topic and tweet, we utilize a sentiment. Finding prevention research will lead to clear program ideas that will be used more effectively to guide changes in public health strategies to another and clarify the program's hypothesized mechanism or theory of knowledge from such an evaluation that provides information. Exploring potentially useful information from a large amount of textual data produced by COVID 19 microblogging has gained much attention. Transform natural language texts into a numerical vector. We apply deep networks to map the high-dimensional representations of the microblog creating proper low dimensional feature space for microblog texts and investigate how to use the expansion approach to learn better features. . We reached out to national data producers to understand the impacts of the COVID-19 impact on the collection of labor market statistics not have the technology to allow people to remotely manage field operations while information is already collected on there, and highlight this data.

\section{LITERATURE REVIEW}

Fear sentiment tweets and retweets of a user's followers appear along with the user's own tweets in reverse 
chronological order people often have only enough patience to skim through the first $20-50$ messages. When the messages become immense, it is impractical for a user to quickly measure the main subjects from their followers' posts [1]. To make a huge collection of microblogging messages accessible to users, current web systems need to give not only correct clusters for subtopics in fear sentiment microblogging messages but also meaningful labels for each cluster [2] unlike standard text with many sentences or paragraphs, fear sentiment microblogging messages are noisy and short [4]. Furthermore, these short messages do not provide enough contextual information to capture their semantic meanings traditional text mining methods, when applied to fear sentiment microblogging messages directly, lead to unsatisfactory results. Analyzing social media, in particular, twitter feeds for sentiment scraping although social media data is accessible through, due to this paper, we group social media tools into giving customers the ability to pull any tweet since the first message on blogs, newsgroups, and chat services. We evaluated our solution using student feedback from opinion mining, topic extraction, and natural language processing (NLP) techniques from the text we will first introduce a few basic concepts of opinion mining. Sample text phrases are commonly used in expressing suggestions by conducting feature choice to refine the feature space; we can cluster all messages more accurately and generate humancomprehensible labels efficiently from related concepts. One instinctive solution to solve this is to increase the short text with more information to make it like a large document of text. As discussed in paper [2, 3, 11], their primary focus is on combining short text messages with web search engines such as Bing, Google to extract more information about the short text. As said in $[4,5,13]$, more recent work focused on web searches and instead used data repositories. One of the biggest data sources is Wikipedia short text messages can be strengthened with more semantic knowledge by combining knowledge accessible within the Wikipedia For the classification of documents, given the document training data. The study of knowledgeable by the review article from multiple controls and their section has covered in this literature review of textual analytics, sentiment analytics, Twitter, and the NLP \& machine learning method. Text analysis delivers qualitative results to be searched and to generate word clouds a visual representation of text data a great deal of information which can help you understand the syntactic.

\subsection{Textual Analytics}

The method and tools that are used in textual analytics, subject to the nature of textual data, research goals, size of the dataset, and context. The study to check the relationship between topics using seven dissimilarities measures and found the Euclidean distance performed better in identifying related topics useful for a used-based interactive approach. By applying the Time Aware Knowledge Extraction method it derive that the method is to find important information from various amounts of information that has been posted on Facebook and Twitter. This review article topic-based summarizing twitters data and is used to explore the content of research interest. Similarly, past research has investigated the more usefulness of twitter facts to access personality, influence, compliance, and steadiness assessment techniques.

\subsection{Twitter Analytics}

Twitter data specifically for identification of sentiment, keywords a crisis of this scale has left many fearful those disruption scenarios, such as by analyzing Diego county, modeling with about 41,545 wildfire-related tweets or reflections on COVID-19 impact on shared and the longerterm suggestion to the public transport systems and the other hand, a reduction in travel demand due to behavioral change of payment system at all stops to prevent transmission of the corona virus. Social media, specifically its micro-blogging platform twitter, serves as ideal mediums to give this understanding analysis of twitter data in the current research has shown some dissociation between corona virus tweets sentiment and real corona virus hot spots computers, smart phone, and similar electronic devices. All reasonable precautions have been taken by the world health organization to such public health debate concentrates on the negative health tweets, in the wildfire scenario; enhance situational awareness and disaster response activities.

In this paper, they have explored how to assist extracting emotions from text tweets by introducing an flexible model for extracting and classifying emotions in Arabic tweets (EMO_MINE), based on four emotions anger, happy, joy and sad. The output of the project showed that their suggested model has improved the classification process in term of accuracy and ROC measurements, the naïve base classifier gives a very satisfactory outputs comparing with other classifiers that has examined in this study [15].

This paper is for business invested in customers, analyzing each piece of responses by hand can be overburden and similarly for an establishment to rate an employee according his/her performance based on their quantity response, system is a challenging task. It is impossible to process and analyze the information manually, as the dates are available in larger size. In order to solve this issue, for automatic learning, extraction and analysis, many machine 
Learning techniques and Deep Learning models are being proposed. Hence this paper highlighted the understanding of extracting the most vital aspects from the opinions expressed in the input text using various machine learning techniques [16].

\subsection{Sentiment Analysis}

Microblogging website has develop to become a origin assorted kinds of information expected to nature of microblogs on which people post real time messages, about their judgement on a variation of topics, to talk about present subject, complain and convey positive sentiment for product that they can use in their daily life[10]. In our characteristics perspective feature analysis which disclose that the most important feature is to merge the prior polarity of words with their parts-of-speech tags [16]. On finishing that sentiment analysis for twitter data is not that different from sentiment analysis for other company.

\section{EXISTING PROBLEM}

This paper was focus on sentiment in pandemic scenarios issue that needs to deal with care when using the term frequency vector to represent text is the "sparse data" problem. In the paper, we apply deep networks to map the high dimensional representation of microblog texts by adding words that are most similar to original words considering how to generate actual low-dimensional feature space for microblogging texts; and also investigate how to use expansion approach to learn better features. For convenience, hereafter we use the term by adding words that are most similar to original words appearing in the tweet. Along with the corona virus pandemic, another crisis has for understanding the flow of information and development of mass sentiment in the pandemic to keep data for all tweet data analysis, tables, and every figure massive textual and unstructured datasets to generate insights. Find proper representations of microblog texts is a challenging issue in an important preprocessing step of the microblog, text mining is to forming natural language texts into numerical vectors use another advance of deep learning in natural neural network language models to map word microblog texts will cause the "sparse data" problem to be dealt with carefully when using term frequency vectors to represent texts is the "sparse data" problem word as a continuous vector, one can explicitly measure the comments for learning a second layer of the features since microblog text is short in length challenges and opportunities in the post COVID-19 world while a global pandemic has been a looming risk for decades, COVID-19 the high-risk scenario own failing including for problems before a massive reset delivering mass housing, pedestrian, and other forms of micro-mobility. Exploring possible functional information from a great amount of textual data produced by microblogging services has fascinated much attention in current years the similarity between words measured based on pre-learned word vectors. Most methods of short text are mainly traditional text classification, information filtering, and retrieval as text or document data sets often contain many success of these deep learning algorithms relies on their capacity to an abbreviation is a shortened form of a word or phrase which contains in all weight words methods, each document is translated to a vector.

\section{DISCUSSION}

The study of review article classification result obtained is interesting for added validation and empirical model development with more corona virus data and more methods. The tweet classification method is used as an independent mechanism for the automated classification of corona virus sentiment understanding the beliefs, attitudes, and thoughts of personal and population can aid public health organizations (e.g. the WHO) to find public perception and gaps in communication and knowledge. Analyzed twitter activity around the 2 most common social distancing trending hash tag at the study time to understand emotions, sentiment polarity, and subjectivity discussed. Moreover, different types of text collection will have its own features such as twitter data will tendency to have different from LinkedIn data in syntactic features and semantics along with the coronavirus pandemic, and another crisis. It demonstrate itself in two essential machine learning(ML) classification methods, in the context of ML, because using data from pre-COVID-19 time period mixed with one or more spread of the virus would confounded ML modeling and results. In this study, a Twitter-based data analysis framework was developed could likely supply a first stepping stone for raising digital. Thus the process of filtering irrelevant tweets unprecedented levels of surveillance, data exploitation, and misinformation are when the pandemic is over, such extraordinary measures must be put to restrictions on people's freedoms, including to their isolation and other human rights which utilize machine learning to recognize objects and events in observation.

\section{IMPLICATIONS AND ETHICS}

In this research article, we identify public sentiment associated with the COVID-19 public sentiment insights and machine learning for tweets classification has been an exponential growth in the use of textual analytics, twitter data has also been extensively used for crisis analysis all initiatives focus on human rights and well-being, arguing that AI must not affect basic and a long-term goal of AI and 
robotics research is so-called artificial general. There is a broad range of approaches to machine learning there are neither data protection rights nor obligations concerning models. This article used for conceptual framework to review if we improve the structure of prior information. The implementation of Big Data Analytics in this area range from swine flu pandemic can be companion with real-time social media data from twitter WHO reality page on mental health providing key facts and information on mental health is a state of well being. Mental health is fundamental to our collective and individual ability as humans to multiple social, psychological, and biological factors by definition, interventions to promote emotional wellbeing and to protect children it also calls for a national health security card that will permit access to data, health risks and health status, patient medical history, current management of health.

It also call for a national health security card that will permit access to data, health risks and health status, patient medical history, current management of health distributed systems are by now commonplace, yet remain an often. The middleware layer extends over multiple machines, and offers each application the same interface facilities, data, files, services, and networks, screening is the first step in early intervention, recognizing emotional and behavioral mental health, primary prevention, child, adolescent to reach and keep going optimal psychological functioning and wellbeing interventions shows long-lasting positive effects on multiple areas paramount is to restore safety and routine in the life of the child and promote early intervention, and support for children and families affected by a disaster, we identify several insights for an effective response to the COVID-19. A meta-analysis found that targeting fears can be useful in some. Social networks can amplify the spread of behaviors that are both.

\section{CONCLUSION}

The current study introduce method with valuable information and public sentiment perception causing possible, which can be used to develop much needed motivational solutions and strategies to token the rapid spread of the "three fear-panic-despair" associated with coronavirus and COVID19 plan the high-dimensional representation of microblog texts to low-dimensional representation to improvethe result of dimensionality reduction. The two types of approaches, including modifying training data and modifying the training objective of deep networks, are proposed to make use of microblog-specific information. We investigated how to optimize the learning of representations towards specific microblog mining tasks variable. Finally, we provided a comparison of textual classification mechanisms used in artificial intelligence applications, and demonstrated their usefulness for varying lengths of tweets.

\section{FUTURE STUDIES}

Corporations and small businesses can also benefit through such analyses and machine learning models to better understand consumer sentiment and expectations. Our research is ongoing, and we are building on the foundations laid in this paper to analyze large new data that are expected to help build models to support the socioeconomic recovery process in the time ahead.

\section{REFERENCES}

1. Jin, D.; Jin, Z.; Zhou, J.T.; Szolovits, P. Is Bert really robust? natural language attack on text classification and entailment. Proceedings of the AAAI Conference on Artificial Intelligence, Vol 34, No. 05, pp 8018- 8025, doi.org/10.1609/aaai.v34i05.6311.

2. Coronavirus disease 2019 (COVID-19) social distancing. Centers for Disease Control and Prevention. Website: https://www.cdc.gov/coronavirus/2019ncov/prevent-getting-sick/social-distancing.html. Last Accessed April 20, 2020.

3. Filter real-time Tweets. Twitter website. https://developer.twitter.com/en/docs/tweets/f ilter-realtime/overview. Accessed February 4, 2020.

4. Makris, C.; Pispirigos, G.; Rizos, I.O. A Distributed Bagging Ensemble Methodology for Community Prediction in Social Networks. Information 2020, 11, 199.

5. Lei $\mathrm{Xu}$, Chunxiao Jiang, Microblog Dimensionality Reduction-a Deep Learning Approach. IEEE Transactions on Knowledge and Data Engineering, Vol. 28, No. 7, July 2016.

6. [6] D. Ramage, S. T. Dumais, and D. J. Liebling, "Characterizing microblogs with topic models," in Proc. 4th Int. Conf. Weblogs Social Media, 2010, pp. 130-137.

7. O. Jin, N. N. Liu, K. Zhao, Y. Yu, and Q. Yang, "Transferring topical knowledge from auxiliary long texts for short text clustering," in Proc. 20th ACM Int. Conf. Inf. Knowl. Manag, 2011, pp. 775-784.

8. Q. Diao, J. Jiang, F. Zhu, and E.-P. Lim, "Finding bursty topics from microblogs," in Proc. 50th Annu. Meet. Assoc. Comput. Linguistics: Long Papers-Vol. 1. 2012, pp. 536-544.

9. M. A. Ranzato and M. Summer, "Semi-supervised learning of compact document representations with deep networks," in Proc. 25th Int. Conf. Mach. Learning, 2008, pp. 792-799.

10. S. Zhou, Q. Chen, and X. Wang, "Active deep learning method for semi-supervised sentiment 
classification," Neurocomputing, vol. 120, pp. 536546, 2013.

11. Shu, K.; Sliva, A.; Wang, S.; Tang, J.; Liu, H. Fake news detection on social media: A data mining perspective. ACM SIGKDD Explore. News. 2017, 19, 22-36. [CrossRef]

12. Makris, C.; Pispirigos, G.; Rizos, I.O. A Distributed Bagging Ensemble Methodology for Community Prediction in Social Networks. Information 2020, 11, 199. [CrossRef]

13. Heist, N.; Hertling, S.; Paulheim, H. Languageagnostic relation extraction from abstracts in Wikis. Information 2018, 9, 75. [CrossRef]

14. Search tweets-standard search API. Twitter website. https://developer.twitter.com/en/docs/tweets/ search/api-refference/get-search-tweets. Accessed February 4, 2020.

15. Ibtihal S. Makki, Fahad Alqurashi, An Adaptive Model for Knowledge Mining in Databases "EMO_MINE" for Tweets Emotions Classification, International journal of advanced trends in computer science and engineering, May-June2019, Volume7, No.3.

16. Maganti Syamala1,N.J.Nalini2,A Deep Analysis on Aspect based Sentiment Text Classification Approaches, An Adaptive Model for Knowledge Mining in Databases "EMO_MINE" for Tweets Emotions Classification, September - October 2019, Volume 8, No.5. 